ENTORNOS, No. 27. | Abril 2014

\title{
Un sistema dinámico discreto en macroeconomía con variables ambientales
}

\author{
A macroeconomics discrete dynamic system \\ with environmental variables
}

\section{Ein diskretes dynamisches system der makroökonomie mit umweltvariablen}

\author{
Mauro Montealegre Cárdenas ${ }^{1}$, Jasmidt Vera Cuenca ${ }^{2}$
}

\begin{abstract}
Resumen
En este texto estudiamos el modelo de generaciones superpuestas, MGS, para describir el comportamiento del crecimiento económico que tiene parámetros y variables exógenas del efecto invernadero para las generaciones futuras; porque estos parámetros y variables afectan la vida económica, y todo el Teorema de Equivalencia Ricardiana no es totalmente comprobable, entonces, el balance de estos sistemas dinámicos puede ser inestable, desaparecer o requerir bifurcaciones del tipo Flip, como por ejemplo, periodos dobles o del tipo Hopf, como los ciclos generativos.
\end{abstract}

Palabras clave: Modelo económico, Sistemas Dinámicos, Bifurcaciones tipo libreta, La dinámica económica con la contaminación.

\begin{abstract}
In this paper we study the model of overlapping generations, MOG, to describe the behavior of the economic growth that has parameters and exogenous variables of the greenhouse effect for future generations; because these variables and parameters affect the economic life of humans, and the whole Ricardian Equivalence Theorem is not totally verifiable. So, the balance of these dynamic systems can be unstable, could disappear or require Flip type bifurcations, ie double periods, or Hopf type, such as generative cycles.
\end{abstract}

Keywords: Economic Model, Overlapping Generations, Dynamical Systems, Bifurcations type Flip, Economic dynamics with pollution.

\section{Zusammenfassung}

In diesem Text studieren wir das Overlapping-Generations-Modell (OLG), um das Verhalten des ökonomischen Wachstums zu beschreiben, welches sich aufrund exogener Parameter und Variablen des Treibhauseffekts auf die zukünftigen Generationen auswirkt. Da diese Parameter und Variablen das ökonomische Leben betreffen und das Ricardianische Äquivalenztheorem nicht vollkommen nachweisbar ist, kann die Balance dieser dynamischen Systeme instabil sein, verschwinden oder Flip-Bifurkationen erfordern, wie zum Beispiel doppelte Perioden oder solche vom Typ Hopf, wie die generativen Zyklen.

\section{Introducción}

El modelo de crecimiento económico de Generaciones superpuestas, MGS, ha recibido especial atención desde la interdisciplinariedad abarcando temas como: la modelización matemática en las Ciencias Sociales, las ecuaciones diferenciales ordinarias, cálculo de variaciones y control óptimo. El estudio de este problema plantea cuestiones relevantes del análisis no lineal que surgen de estudiar las interrelaciones de la actividad económica del hombre con las complejidades de la naturaleza.
Las primeras publicaciones sobre este tema fueron hechas por Paul Samuelson en 1958, y Peter Diamond en 1965. El modelo básico de Generaciones Superpuestas, MGS, tiene las siguientes características: las personas viven durante dos períodos, en el primer período de la vida se conoce como periodo joven, el segundo período de la vida como período Antiguo. El modelo MGS comparte las siguientes hipótesis básicas:

1 - (mmonteal@usco.edu.co) Director Grupo de investigación DINUSCO. Universidad Surcolombiana.

2 - (jveracuenca@hotmail.com) Integrante Grupo de investigación DINUSCO. Universidad Surcolombiana. 
i) Los individuos reciben una dotación económica de bienes en el nacimiento,

ii) El capital que se genera en el primer periodo de la vida se consume en el segundo periodo,

iii) El valor del dinero permanece constante,

iv) Las personas deben consumir en todos los períodos y hay una función de consumo conocida.

La dinámica con estas hipótesis es hiperbólica, es decir, con equilibrios son estables, pero al no observar la hipótesis ii) como en A. Medio 1992, la dinámica puede ser no - hiperbólica, esto es estructuralmente inestable, siguiendo esta dirección estamos delineando situaciones más complejas debilitando algunas de las hipótesis de i -iv de esta introducción.

En el modelo MGS pueden aparecer ciclos estables o inestables. Estos ciclos son detectados utilizando el Teorema de Bifurcación de Hopf, donde el parámetro de bifurcación es puramente tecnológico. Estos ciclos endógenos en torno de un equilibrio fueron descubiertos por J.M. Grandmont en 1985; también se ha demostrado que al involucrar variables ambientales puede surgir en la frontera inestabilidad en torno de ciertos equilibrios que constituye una bifurcación Flip, esto es, duplicación de periodos para equilibrios del sistema antropoatmosferico. Por ello este estudio es útil para visualizar lo que puede pasar en la vida socio ambiental de futuras generaciones.

En el trabajo de P. Reichlin (2006) se muestra que el resultado de Grandmont depende de una tecnología muy simple, es decir, aquella en la que el trabajo actual es el único insumo necesario para producir el desarrollo económico. Esta dinámica económica aún es más compleja cuando se involucra el efecto de los gases de invernadero, alta concentración de $\mathrm{CO}_{2}$ en la atmosfera, como lo plantean entre otros Dong Cao, L. Wang y Y. Wang (2011).

Sobre la importancia de este tipo de estudios han llamado la atención Stern (2006), quien prueba que se deben tomar acciones decisivas para reducir el efecto invernadero para evitar grandes catástrofes con impredecibles costos socio-económico. De otra manera las futuras generaciones sufrirán grandes afectaciones muchos mayores que el costo de evitar la emisión descontrolada de gases de invernadero a la atmosfera, que es nuestra casa. Por ello el foro en Económico Mundial, 2014, el nuevo modelo económico debe dar valor a las externalidades negativas, daño ambiental, teniendo en cuenta la capacidad de carga del planeta para asegurar a las futuras generaciones vida sostenible e inclusiva.

El aporte específico de este artículo consiste en reducir el cálculo a la variedad central en torno a un punto de equilibrio cuyo desdoblamiento se debe a una bifurcación de tipo Flip o Hopf, éste es un resultado del proyecto de investigación de la Universidad Surcolombiana denominado "Bifurcación de Modelos Económicos de Generaciones Superpuestas".

\section{Marco Teórico Básico}

\subsection{Un Sistema Dinámico Discreto Unidimensional Sin Polución}

Según P.A. Diamond 1965, la optimizando la utilidad $w$ con dos restricciones, una con respecto a los ingresos laborales de un trabajador y otra con respecto al ahorro en el siguiente modelo, MGS,

$$
\left\{\begin{array}{c}
\max _{C_{1 t}} W=u\left(c_{i t}\right)+(1+\theta)^{2} u\left(c_{2 t+1}, t+1\right) \\
c_{1 t}+s_{t} \leq w_{t} \\
c_{2 t+1} \leq\left(1+E\left(r_{t+1}\right)\right) s_{t} \\
c, w, u, r \in \mathbb{R}^{+} \quad s, \theta \in \mathbb{R}_{0}^{+}
\end{array}\right.
$$

donde $c_{1 t}, c_{2 t}$ son los consumos (o utilidades) en el periodo Joven $t$ y el periodo de retiro $t+1 ; w_{t}$ salario en el periodo $t ; s_{t}$ es ahorro en el periodo $t ; r_{t+1}$ tasa de interés por los préstamos en un periodo $t+1$; la esperanza de valor capital es constante,

$E\left(r_{t+1}\right)=r_{t+1}, y \lim _{K_{t} \rightarrow \infty} \frac{K_{t}}{K_{t+1}}=(1+\theta)^{-1}$

es el factor de discontinuidad intersubjectiva para utilidades futuras.

El modelo (1) tiene soluciones óptimas

$c_{t+1}^{*}, s_{t+1}^{*}=s_{t}^{*}\left(w_{t} ; r_{t+1}\right) ; \quad$ el ahorro total es

$s_{t}=N_{t} s_{t}$ donde $N_{t}=N_{0}(1+n)^{t}$ con $-1<n<1$.

La producción total $\boldsymbol{y} t$ depende del capital $\boldsymbol{K} \boldsymbol{t}$ y del trabajo $\boldsymbol{N} t$ mediante una función $\boldsymbol{F}$ homogénea de primer grado, esto es,

$$
\left\{\begin{array}{l}
y_{t}=F\left(k_{t}, N_{t}\right), \quad N_{t} \in \mathbb{R}^{+}, \quad k_{t} \in \mathbb{R}_{0}^{+} \\
\frac{y_{t}}{N_{t}}=y_{t}=f\left(k_{t}\right)
\end{array}\right.
$$

donde $K \boldsymbol{t}$ y $\boldsymbol{Y} t$ es la inversión per-cápita y producción per carpita, respectivamente; tal que $f$ independientemente de depreciación y satisface las condiciones de Ineda que se ilustra en la Figura 1:

$$
\begin{aligned}
& f^{\prime}\left(k_{t}\right)>0, f^{\prime \prime}\left(k_{t}\right)>0, f(0)=0, \\
& \lim _{k \rightarrow 0} f^{\prime}\left(k_{t}\right)=\infty, \lim _{k \rightarrow \infty} f^{\prime}\left(k_{t}\right)=0 .
\end{aligned}
$$




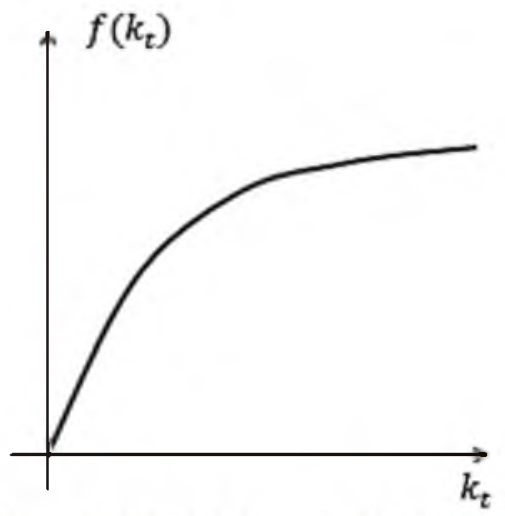

Figura 1. Condiciones de Ineda para $f$

Básicamente el modelo MGS de Diamond optimiza el beneficio máximo $\boldsymbol{P}$ sujeto

$$
\left\{\begin{array}{l}
\max _{N_{t}, k_{t}} P=y_{t}-w_{t} N_{t}-r_{t} K_{t} \\
y_{t} \leq F\left(K_{t}, N_{t}\right)
\end{array}\right.
$$

cuyo niveles óptimos para los precios del capital y el trabajo son equivalentes a sus productos marginales, para la mano de obra y el capital,

$$
\left\{\begin{array}{l}
M P_{N}=w_{t}=f\left(k_{t}\right)-K_{t} f^{\prime}\left(k_{t}\right) \\
M P_{k}=r_{t}=f^{\prime}\left(K_{t}\right)
\end{array}\right.
$$

Para simplificar el modelo asumimos que hay un único bien en el mercado, y de la hipótesis i) - iv) surge la siguiente identidad iguala la inversión se iguala al ahorro entre estas generaciones,

$$
k_{t+1}-k_{t}=N_{t} s_{t}-k_{t}
$$

El lado derecho de (6) establece que el ahorro neto es la diferencia entre el ahorro de los trabajadores y el desahorro de los pensionados, entonces la ecuación para la dinámica del capital, dependiente de la solución optima $s$ de (1), tiene la siguiente expresión formal:

$$
k_{t+1}=\frac{s_{t}^{*}\left(w_{t}, r_{t+1}\right)}{1+n}
$$

remplazando en (7) los precios de los factores de equilibrio (5) obtenemos el siguiente mapeo

$k_{t+1}=G\left(k_{t}\right)$,

$k_{t+1}=\frac{s^{*}\left(\left[f\left(k_{t}\right)-K_{t} f^{\prime}\left(k_{t}\right)\right],\left[f^{\prime}\left(k_{t}\right)\right]\right)}{1+n}$.

La ecuación (8) es conocida como el ahorro focalizado en este modelo. La función de utilidad es estrictamente cóncava e isoelástica, por ello podemos asumir que $u(\cdot)=\ln (\cdot)$, la cual garantiza la dinámica futura y la cantidad optima de consumo y ahorros son los siguientes:

$$
\left\{\begin{array}{l}
c_{1 t}^{*}=\left(\frac{1+\theta}{2+\theta}\right) w_{t} \\
s_{t}^{*}\left(w_{t}\right)=w_{t}-c_{1 t}^{*}=\left(\frac{1}{2+\theta}\right) w_{t}
\end{array}\right.
$$

donde la tasa de interés futura no afecta la escogencia del consumo y el ahorro óptimo es simplemente proporcional a los ingresos individuales. Notamos que si los valores de utilidad son tales que $\theta=0$, la mitad de los salarios son ahorros para el segundo periodo; y si estas generaciones asumen que el futuro no importa, $\theta \rightarrow \infty$, entonces $s_{t}^{*} \rightarrow 0$.

La función de producción en (2) es una potencia homogénea de primer grado, ver P. Reichlin 2006, como la siguiente:

$$
\left\{\begin{array}{l}
Y_{t}=F\left(K_{t}, N_{t}\right)=K_{t}^{\alpha} \cdot N_{t}^{1-\alpha}, \\
0<\alpha<1, N \in \mathbb{R}^{+}, K \in \mathbb{R}_{0}^{+} \\
\frac{y_{t}}{N_{t}}=f\left(k_{t}\right)=k_{t}^{\alpha} .
\end{array}\right.
$$

Sustituyendo $f$ de (10) en (5) obtenemos el siguiente proceso dinámico para los precios de los factores de los salarios y capitalización,

$$
\left\{\begin{array}{l}
w_{t=(1-\alpha) k_{t}^{\alpha}} \\
r_{t}=\alpha k_{t}^{\alpha-1}
\end{array}\right.
$$

Sustituyendo también el ahorro óptimo (9) en (8), obtenemos el siguiente sistema dinámico unidimensional,

$$
k_{t+1}=\sigma k_{t}^{\alpha}, \quad \operatorname{con} \sigma=\frac{1-\alpha}{(1+n)(2+\theta)} .
$$

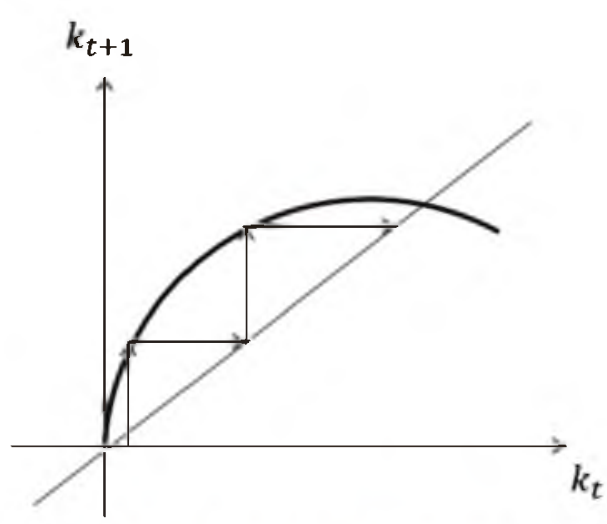

Figura 2. Dinámica de (12) 
El comportamiento local, esto es, en torno de sus equilibrios, son determinados por los autovalores del modelo linealizado; es simplemente la derivada de $k_{t+1}$ con respecto a $k_{t}$ calculada en el equilibrio trivial,

$$
\begin{gathered}
\bar{k}_{1}=0,\left.\quad \frac{d k_{t+1}}{d k_{t}}\right|_{\bar{k}_{1}}=+\infty, \\
\bar{k}_{2}=\sigma^{\frac{1}{1-\alpha}},\left.\quad \frac{d k_{t+1}}{d k_{t}}\right|_{\bar{k}_{2}}=\alpha .
\end{gathered}
$$

El equilibrio trivial es repulsor y satisface las hipótesis sobre los parámetros, el equilibrio $\bar{k}_{2}$ atrae las demás trayectorias con convergencia monótona. No hay cambio en la estabilidad por los cambios parciales de los parámetros en los estados de reposo, esto es,

$$
\frac{\partial \bar{k}_{2}}{\partial n}<0, \quad \frac{\partial \bar{k}_{2}}{\partial \theta}<0, \quad \frac{\partial \bar{k}_{2}}{\partial \alpha}<0 .
$$

El efecto de la variación del parámetro de producción $\alpha$, elasticidad de la producción con respecto al capital, puede resultar desconcertante. Sin embargo en el modelo de Diamond la totalidad del capital representado en la producción es pagada con interés a los jubilados, quienes usan todos estos recursos en el consumo. La formación de capital en esta economía se deriva completamente del ahorro sobre el salario de los trabajadores, así que es lógico que mientras la economía tenga más avances tecnológicos ( $\alpha$ grande) tiene el estado de equilibrio per cápita de más bajo.

\subsection{Un Sistema Dinámico Unidimensional Discreto Con Polución}

De A. Medio (1992) podemos asumir dos tipos de procesos de captura de la polución por la atmosfera:

a) De captura proporcional a la emisión de poluentes,

$$
A_{t+1}=\frac{A_{t}^{2}}{\hat{A}}+a k_{t}-b d_{t}
$$

donde $\boldsymbol{A}_{\boldsymbol{t}}$ es el índice de polución en el servicio $\boldsymbol{t}, \boldsymbol{d}_{\boldsymbol{t}}$ es el trabajo relacionado con el control de la contaminación, $\boldsymbol{a}>\boldsymbol{0}$ es la razón relacionada con el crecimiento económico, $\boldsymbol{b}>\boldsymbol{0}$ es el coeficiente de control tecnológico de la contaminación; y planeamos el siguiente problema de optimización:

$$
\left\{\begin{array}{c}
\max _{c_{t}}\left\{c_{t+1}-\frac{A_{t}^{2}+1}{2 B}\right\} ; B>0 \\
\text { Sujeto a: } \quad k_{t+1}+d_{t}=w_{t} \\
c_{t+1}=r_{t} k_{t+1}
\end{array}\right.
$$

Por el método de multiplicadores de Lagrange encontramos que la condición de primer orden para (16) es

$$
r_{t+1}=\frac{A_{t+1} b}{B}
$$

Entonces teniendo en cuenta (15), (16), (17) y (5) resulta el siguiente sistema dinámico,

$$
\begin{aligned}
& B \frac{f\left(k_{t+1}\right)}{b}-b k_{t+1} \\
& =B^{2} \frac{\left[f^{\prime}\left(k_{t}\right)\right]^{2}}{b^{2} \hat{A}}+a k_{t}-b\left[f\left(k_{t}\right)-k_{t} f^{\prime}\left(k_{t}\right)\right]
\end{aligned}
$$

asumiendo que la función de producción es

$$
\begin{aligned}
& f\left(k_{t}\right)=k_{t} r\left(k_{t}\right)+w\left(k_{t}\right) \text { y }(18) \quad \text { es tal que } \\
& k_{t+1}=k_{t} \text { y } k_{t+1}=G\left(k_{t}\right)
\end{aligned}
$$

Resolviendo y estudiando su estabilidad en torno de $k=1$ a través de la siguiente ecuación variacional,

$$
\frac{d k_{t+1}}{k_{t}}=\lambda
$$

resulta que: $|\lambda|<1, k=1$ es estable; $\lambda=-1$ en $k=1$ hay una bifurcación de tipo Flip, esto es

$G^{2}\left(k_{t}\right)=k_{t}, \operatorname{con} D G^{2}(1)=1$.

b) De captura de polución consideramos un umbral crítico.

En este caso consideramos una función de producción de tipo logístico como la siguiente,

$$
f\left(k_{t}\right)=b k_{t}^{\beta}\left(m-k_{t}\right)^{r}, k_{t} \leq m,
$$

donde podemos asumir que $\beta=r=1$ y para despejar un proceso dinámico complejo, entonces resulta que (18) es el siguiente sistema dinámico discreto:

$$
k_{t+1}=M k_{t}^{\beta}\left(m-k_{t}\right)^{r}
$$

Para (21) encontramos su punto critico $k_{c}$ que resuelva la siguiente ecuación,

$$
\begin{gathered}
\frac{d k_{t+1}}{d k_{t}}= \\
M\left[\beta k_{t}^{\beta-1}\left(n-k_{t}\right)^{r}-r k_{t}^{\beta}\left(m-k_{t}\right)^{r-1}\right]=0,
\end{gathered}
$$


$k^{b}=f^{-1}\left(k^{c}\right)$ y $k^{*}$ es el punto fijo de $f$, esto es $f\left(k^{*}\right)=k^{*}$, podemos asumir que $k^{c}<k^{*}$.

En el caso de que $k^{b}<k^{*}$ se tiene que $k^{b}<k^{c}$. De otro lado sea $k^{m}$ la máxima cantidad de capital alcanzable en este proceso, esto es,

$$
f\left(k^{c}\right)=M\left(k^{c}\right)^{\beta}\left(m-k^{c}\right)^{r}=k^{m}
$$

Resultando que $f\left(k^{m}\right)=0$ y por ello, tal como se ilustra la siguiente grafica de (21),

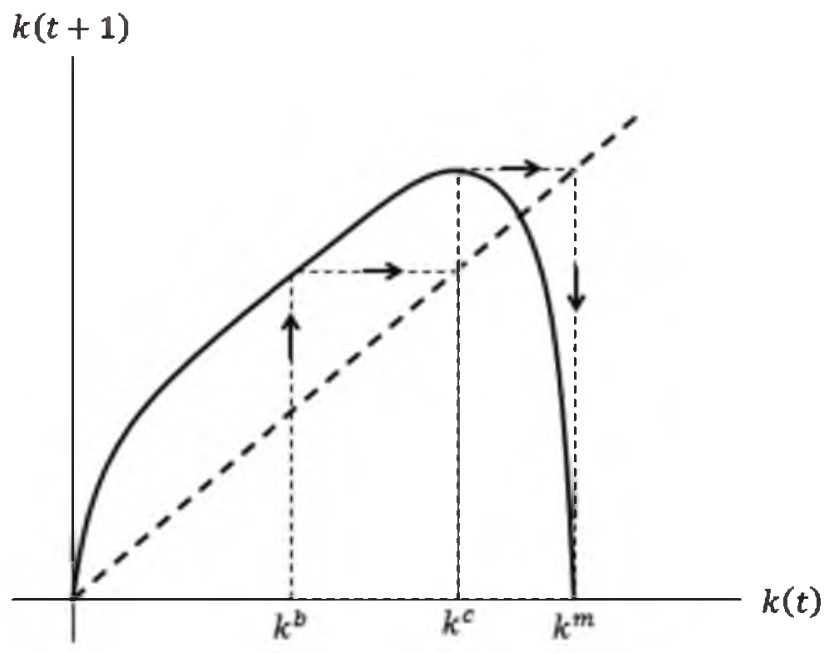

Figura 3. Crecimiento de la polución con umbral

de lo anterior tenemos que,

$$
\begin{gathered}
0<k^{b}<k^{c}<k^{m} \quad \mathrm{y} \\
f^{3}\left(k^{b}\right)<k^{b}<f\left(k^{b}\right)<f^{2}\left(k^{b}\right),
\end{gathered}
$$

y por el Teorema de Li - Yorje, resulta que el proceso (23) es caótico.

\section{Hacia un método multidimensional y dinámica con externalidades ambientales}

\subsection{El Problema con aportes pensionales}

Ahora consideremos el problema (1) sustituyendo las siguientes restricciones de Blanchard y Fischer (1989),

$$
\begin{gathered}
c_{1 t}+s_{t}+d_{t} \leq w_{t} \\
c_{2 t+1} \leq\left(1+E_{t}\left(r_{t+1}\right)\right) s_{t}+(1+n) E_{t}\left(d_{t+1}\right)
\end{gathered}
$$

donde $d_{t+1}$ es el pago a la seguridad social en el periodo $t+1$ (lo mismo para $d_{t}$ ); con plena precisión del rendimiento de los fondos pensionales

$E_{t}\left(d_{t+1}\right)=d_{t+1}, d \in \mathbb{R}_{0}^{+}$

y se supone $d_{t}=\gamma w_{t}$, además con la función de producción de (10) y de(11) obtenemos,

$$
d_{t}=\gamma(1-\alpha) k_{t}^{\alpha} .
$$

Entonces el problema de optimación (1) con las restricciones (24) y asumiendo que la función de utilidad es el logaritmo natural se obtiene el siguiente consumo óptimo:

$c_{1 t}^{*}=\frac{1+\theta}{2+\theta}\left[w_{t}-\gamma w_{t}+\gamma \frac{1+n}{1+r_{t+1}} w_{t+1}\right]$.

En este modelo se requiere que los agentes tengan perfecta previsión del salario futuro, esto es, en cuento al trabajo marginal y capital marginal. Observamos que si $r_{t+1}=n$, entonces (26) es igual a (9) lo que significa que en este modelo el consumo óptimo es neutral con respecto al esquema de pensión en el estado de equilibrio.

El ahorro óptimo (9), teniendo en cuenta las contribuciones presentes y futuras es el siguiente:

$s_{t}^{*}=\frac{1}{2+\theta} w_{t}-\frac{1}{2+\theta} d_{t}-\frac{(1+n)(1+\theta)}{\left(1+r_{t+1}\right)(2+\theta)} d_{t+1}$

en el caso neutral $n=r_{t+1}$ el estado de equilibrio del ahorro $\bar{s}=\frac{\bar{w}}{2+\theta}-\bar{d}$ por ello $\frac{\partial \bar{s}}{\partial \bar{d}}=-1$, lo que

significa que los incrementos para la seguridad social es contrabalanceado por el mismo decrecimiento en el ahorro.

En este modelo se reduce el ahorro a través de dos efectos como se observa en (27): en el segundo término por la reducción de los pagos y la parte discontinua de los pagos que son transferencias a los que normalmente se retiran; el tercer término representa el efecto sobre el ahorro debido a las contribuciones de los próximos consumos de las próximas generaciones y es la misma que la proporción de impuestos sobre la nómina del consumo corriente $\underline{1+\theta}$, llevada a valor presente $E_{t}\left(r_{t+1}\right)$.

$$
\overline{2+\theta}
$$

El tamaño $(n+1)$ de la transferencia devengado por un único agente depende del número de agentes que contribuyen en $(t+1)$ con respecto al número de agentes que se retiran en $(t+1)$, esto es, en economías con poblaciones declinantes o tasas de interés muy altos, los agentes tienen que ahorrar más para asegurar los fondos de retiro óptimos.

Los efectos de los parámetros sobre el ahorro total óptimo son los siguientes:

$\frac{\partial s^{*}}{\partial n}<0, \frac{\partial s^{*}}{\partial r_{t+1}}>0, \frac{\partial s^{*}}{\partial \theta}<0, \frac{\partial s^{*}}{\partial \gamma}<0$. 
Como la formación de capital en este modelo es financiado por el ahorro de los trabajadores, los valores de acumulación y el estado de reposo del capital necesariamente disminuyen.

\subsection{Cálculo de Equilibrio y su Estabilidad}

Como en (7) teniendo en cuenta la deducción sobre la nómina obtenemos,

$$
k_{t+1}=\frac{\left(s_{t}^{*}+d_{t}\right)}{(1+n)}
$$

Sustituyendo el ahorro optimo, deducciones de nómina y beneficios futuros, obtenemos

$$
\begin{array}{r}
k_{t+1}=\frac{(1-r)}{(2+\theta)(1+n)} w_{t}- \\
\gamma \frac{(1+\theta)}{\left(1+r_{t+1}\right)(2+\theta)} w_{t+1} .
\end{array}
$$

Sustituyendo los valores del beneficio máximo para precios (26) se obtiene la siguiente relación implícita en lugar de la relación explicita de (12),

$$
\begin{aligned}
& k_{t}^{\alpha}(1-\alpha)(1-r)\left(1+\alpha k_{t+1}^{\alpha-1}\right) \\
& \quad=(t+n)\left[k_{t+1}(2+\theta)+\right. \\
& \left.\quad k_{t+1}^{\alpha}(\alpha(2+\theta)+r(1-\alpha)(1+\theta))\right]
\end{aligned}
$$

de la cual deducimos que la ecuación para la dinámica inversa es la siguiente:

$k_{t}=\left\{\frac{(1+n)\left(k_{t+1}(2+\theta)+k_{t+1}^{\alpha}[\alpha(2+\theta)+r(1-\alpha)(1+\theta)]\right)}{(1-\alpha)(1-r)\left(1+\alpha k_{t+1}^{\alpha-1}\right)}\right\}^{\frac{1}{\alpha}}$.

Entonces los equilibrios son: $\bar{k}_{1}=0$, y la solución de

$$
\begin{aligned}
& -(\rho \sigma)^{-1} \bar{k}^{2 \alpha-2}+ \\
& \left(\alpha+r(1+n)(1-\alpha)(1+\theta) \rho^{-1}\right. \\
& \left.-(\alpha \rho \sigma)^{-1}\right) \sigma \bar{k}^{\alpha-1}+1=0
\end{aligned}
$$

donde

$$
\rho=[\alpha(1-\alpha)(1-r)]^{-1}, \sigma=[(1+n)(2+\theta)]
$$

y $\rho>\sigma>0$.

Haciendo $z=\bar{k}^{\alpha-1}$, observamos que $\alpha-1<0$ y obtenemos el siguiente polinomio,

$$
\begin{array}{r}
z^{2}-(\alpha \rho \sigma+r(1+n)(1+\alpha) \\
\left.(1+\theta) \rho-\alpha^{-1}\right) z-\rho \sigma=0
\end{array}
$$

cuyas raíces $z_{2}$ y $z_{3}$ están relacionadas en la siguiente fórmula,

$$
\bar{k}_{2,3}=\left(\frac{1}{z_{2,3}}\right)^{\frac{1}{1-\alpha}}
$$

Observamos que el discriminante de (33) es no negativo, por ello del teorema Descartes aseguramos que hay dos raíces reales, una positiva y una negativa,

$z_{2}>0$ y $z_{3}<0$; el tercer estado de reposo es

$\bar{k}_{3}=\left(\frac{1}{z_{3}}\right)^{\alpha-1}$ el cual puede ser real o complejo,

dependiendo de $\alpha$ y es imposible estudiar la dinámica en torno de este equilibrio, porque depende de si $\frac{1}{1-\alpha}$
es par o impar.

Por otro lado, encontramos los efectos sobre el estado de reposo $\bar{k}_{2}$ de los parámetros teniendo en cuenta los rangos permitidos, son:

$\frac{\partial \bar{k}_{2}}{\partial n}<0, \frac{\partial \bar{k}_{2}}{\partial \theta}<0, \frac{\partial \bar{k}_{2}}{\partial \alpha}<0, \frac{\partial \bar{k}_{2}}{\partial \gamma}<0$,

tal como se esperaba, al variar el parámetro de producción, alta tasa de crecimiento poblacional, altas ratas de discontinuidad y altas tasas de impuestos sobre la nómina, induce bajos valores de estado de reposo para capital per-cápita.

El autovalor de la dinámica futura es el inverso del autovalor de (32), porque la relación entre $k_{t}$ y $k_{t+1}$ es uno a uno, y además $\left.\frac{\partial k_{t+1}}{\partial k}\right|_{\bar{k}_{1}^{-}}=+\infty$

esto es, $\overline{k_{1}}$ es un repulsor; y para un rango de parámetros aceptable se tiene que

$$
\left.\frac{\partial k_{t+1}}{\partial k_{t}}\right|_{\overline{k_{2}}}
$$

es monótona, luego $\overline{k_{2}}$ es un atractor local, por ello se tiene que en el cuadrante $\left(k_{t}, k_{t+1}\right)$ el único estado de reposo es $\bar{k}_{2}$ dependiendo de la evolución inicial $k_{0}:{ }^{\text {si }} k_{0}<\bar{k}_{2}$ es atractor, $k_{0}>\bar{k}_{2}$ es repulsor.

\subsection{Problema De Optimización Con La Externalidad Causada Por La Polución}

El modelo con ocio estudiado por Medio (1992), los jóvenes difieren del consumo, $v_{1}\left(l_{t}\right)$ es la desutilidad donde $l_{t}$ es la parte de este trabajo gastado en formación durante el primer periodo de la vida; $u\left(c_{t+1}\right)$ es la utilidad del consumo en el segundo periodo de la vida, $R_{t}=1+r_{t}$ es el factor de interés para un solo bien.

En este caso la condición de equilibrio es más compleja, 


$$
k_{t+1}-k_{t}=y_{t}-c_{1 t}-c_{2 t}
$$

donde $c_{2 t}=s_{t-1}$ es el desahorro de los jubilados, y para función de producción usamos el Teorema de Euler para funciones homogénea de primer orden, (36) se convierte en, $k_{t+1}-k_{t}$

$$
\begin{aligned}
& =r_{t} k_{t}+W_{t} N_{t}-N_{t} c_{t}-\left(1-r_{t}\right) N_{t-1} s_{t-1} \\
& =N_{t} s_{t}+r_{t} k_{t}-\left(1+r_{t}\right) N_{t-1} s_{t-1} .
\end{aligned}
$$

En este caso los jóvenes en el periodo " $t$ " enfrentan el siguiente problema de optimización:

$$
\left\{\begin{array}{c}
\max _{c_{t+1}, l_{t}} W=u\left(c_{t+1}\right)-v\left(l_{t}\right) \\
\text { Sujeto a, } \\
k_{t} \leq w_{t} l_{t} \\
c_{t+1} \leq R_{t} k_{t} \\
c, w, u, v, R, l \in \mathbb{R}^{+}, k \in \mathbb{R}_{0}^{+} s_{t}=k_{t}
\end{array}\right.
$$

donde $u($.$) Y v($.$) son continuas en [0, \infty]$ con $u^{\prime}\left(c_{t+1}\right)>0$ y $u^{\prime \prime}\left(c_{t+1}\right)<0 ; v^{\prime}\left(l_{t}\right)>0$, $v^{\prime \prime}\left(l_{t}\right)>0, \lim _{l_{t} \rightarrow \infty} v^{\prime}\left(l_{t}\right)=+\infty, \lim _{l_{t} \rightarrow 0} v^{\prime}\left(l_{t}\right)=0$. La condición de primer orden para el problema (32) es,

$$
\left\{\begin{array}{c}
u^{\prime}\left(c_{t+1}\right) \frac{\partial c_{t+1}}{\partial u_{t}}=v^{\prime}\left(l_{t}\right) \\
c_{t+1}=R_{t+1} w_{t} l_{t}
\end{array}\right.
$$

Sustituyendo la primera restricción de (38) en la primera ecuación de (39), tenemos la condición necesaria y suficiente para la existencia de la solución óptima $\left\{c^{*}, l^{*}\right\}_{t}$ donde

$$
\begin{aligned}
& \mathcal{U}^{\prime}\left(c_{t+1}\right)=\mathcal{v}\left(l_{t}\right), \quad \mathcal{u}\left(c_{t+1}\right) \\
& =c_{t+1} u^{\prime}\left(c_{t+1}\right), v\left(l_{t}\right)=l_{t} v^{\prime}\left(l_{t}\right),
\end{aligned}
$$

si $U^{-1}$ existe la dinámica futura es determinada por $f$ definido así,

$$
\left\{\begin{array}{c}
c_{t+1}^{*}=f\left(l_{t}^{*}\right) \\
f=\mathcal{U}^{-1}\left[\mathcal{V}\left(l_{t}\right)\right]
\end{array}\right.
$$

en particular suponemos que la relación de tecnología es del tipo Leontief con una labor $l_{t}$ aplicado en un periodo previo, $y_{t}=\min \left[l_{t}, b k_{t+1}\right] \quad$; entonces asumiendo el pleno empleo de los factores se tiene $l_{t+1}=l_{t}=b k_{t-1}$
Como la función de utilidad es iso - elástica, así como la disualidad, podemos asumir que,

$$
\left\{\begin{array}{c}
u\left(c_{t+1}\right)=\frac{c_{t+1}^{\alpha}}{\alpha}, \quad 0<\alpha<1 \\
v\left(l_{t+1}\right)=\frac{l_{t}^{\beta}}{\beta}, \quad \beta>1
\end{array}\right.
$$

de (39), (40) y (41) obtenemos que

$$
c_{t+1}=l_{t}^{\frac{\beta}{\alpha}} \text {. }
$$

Entonces la dinámica futura de este modelo económico, se determina por el siguiente sistema,

$$
\left\{\begin{array}{c}
c_{t+1}=l_{t}^{\frac{\beta}{k}} \\
l_{t+1}=b\left(l_{t}-c_{t}\right)
\end{array}\right.
$$

donde (43) tiene dos estados de equilibrios, $E_{1}=(0,0)$ y $E_{2}$ es el primer cuadrante.

El linealizado de (43) en $E_{2}$ es,

$$
J=\left(\begin{array}{cc}
0 & \frac{\beta(b-1)}{\alpha b} \\
-b & b
\end{array}\right)
$$

cuya ecuación característica es

$\lambda^{2}-\operatorname{Traza}(J) \lambda+\operatorname{det}(J)=0$

entonces para que $\left|\lambda_{1}\right|<1$ y $\left|\lambda_{2}\right|<1$

y se tiene las siguientes condiciones:

$$
\begin{aligned}
1+\operatorname{Traza}(J)+\operatorname{det}(J) & >0 \\
1+\operatorname{Traza}(J)+\operatorname{det}(J) & <0 \\
1-\operatorname{det}(J) & >0
\end{aligned}
$$

Si (i) es igual a cero, $\lambda_{1}=-1$, es una bifurcación tipo Flip; es una bifurcación tipo Fold si (ii) es igual a cero; es bifurcación del tipo Neimar - Sacker si $\operatorname{det}(J)=1$

De los valores de parámetros para (43) la única condición que puede fallar es (iii).

Si $\mu=\frac{\beta}{\alpha}$ y $\mu b-\mu-1>0$, entonces $E_{1}$ es estable; del Jacobiano en $E_{2}$ con $\operatorname{det} J=1$, analizamos la bifurcación de este equilibrio en las regiones $(b, k)$ delimitada por las curvas $h_{1}$ y $h_{2}$ :

$$
\begin{aligned}
& h_{1}(b, \mu)=\mu b-\mu-1=0, \\
& h_{2}(b, \mu)=\frac{b^{2}}{b-1}-4 \mu=0,
\end{aligned}
$$

donde $h_{2}$ es la curva que corresponde el cambio de valores propios de reales a complejos. 


\section{Sistemas dinámicos con la externalidad de la polución}

Pero la utilidad derivada del consumo en el segundo periodo $c_{t+1}$ es inseparable del índice de concentración del gas invernadero $\mathrm{CO}_{2}, A_{t}, \mathrm{D}$. caos y otros 2011, por ello en el problema (38) se debe maximizar a $u\left(c_{t+1}, A_{t}\right)-v\left(l_{t}\right)$.

A fin de evitar una complicación excesiva, supondremos que sólo existe un bien, el cual se puede

consumir o acumular en forma de capital y la tecnología empleada se describe con la siguiente función de producción del tipo Cobb-Douglas,

$$
y_{t}=R K_{t}^{\alpha}\left(A_{t} L_{t}\right)^{1-\alpha} .
$$

Con respecto al nivel preindustrial $k$; la interdependencia de la utilidad del consumo $u\left(c_{t+1}\right)$ con respecto $A_{t}$ es formalizado por la variable de "la razón de discontinuidad" $\theta\left(A_{t}\right)=\frac{A_{t}}{k}, k$, entonces,

$$
1+\theta\left(A_{t}\right)
$$

representa el múltiplo del nivel pre-industrial, con medida común del cambio de la duplicación de la conservación en el cambio climático, que es $\log _{2}\left(1+\theta\left(A_{t}\right)\right)$

el efecto individual en este problema de optimización se incorpora tomando el reciproco del factor de discontinuidad,

$$
\frac{u\left(c_{t+1}\right)}{1+\theta\left(A_{t}\right)}
$$

Según Conwey (2003) después de duplicar el nivel preindustrial de $\mathrm{CO}_{2}$ la atmosfera no puede adsorber las emisiones; para $k=280$ partes por millón el límite regulable de $A_{t}$ es $\mathbf{1 0 0}$ el factor de discontinuidad ambiental tiene el siguiente rango

$$
0.25 \leq(1+\theta)^{-1} \leq 1 .
$$

El índice ambiental entra en el problema de optimización a través del factor de discontinuidad, entonces de (40) y si $U^{-1}$ existe, tenemos

$$
\left\{\begin{array}{c}
\frac{U\left(c_{t+1}\right)}{1+\theta\left(A_{t}\right)}=v\left(l_{t}\right), c_{t+1}^{*}=f\left(l_{t}^{*}, A_{t}\right), \\
f=\mathcal{U}^{-1}\left[\left(1+\theta\left(A_{t}\right)\right) \mathcal{v}\left(l_{t}\right)\right] .
\end{array}\right.
$$

La unidad laboral per-cápita para $l_{t}$ y como la producción requiere la entrada de un factor de energía representado por las emisiones $Z_{t}$, conocida como la producción de externalidades la cual depende de la inversa del periodo anterior, $b k_{t+1}$. La producción per-capita se aproxima por la siguiente relación de Leontief,

$$
x_{t}=\min \left[l_{t}, b k_{t-1}, \rho z_{t}\right] \text {, }
$$

donde las constantes referidas a procesos de producción a corto plazo; $1, b, \rho$ son respectivamente: la producción total per-cápita por unidad de labor, unidad de capital y contaminación $\rho>0$. Este último parámetro indica que tan "verde" es la producción tecnológica y juega su papel importante en la existencia del equilibrio sostenible

$$
x_{t}=l_{t}=b k_{t-1}=\rho z_{t},
$$

de las dos expresiones intermedias resulta que

$$
l_{t+1}=b\left(l_{t}-c_{t}\right),
$$

donde estamos asumiendo que: el mercado es perfectamente competitivo, los factores de producción reciben sus productos marginales, y el capital se acumula solamente en un periodo.

Con respecto a la emisión de contaminación, al principio de cada periodo el nivel adicional de emisiones acumuladas es equivalente al "stock" el cual no ha sido captado por el sumidero natural, más las emisiones corrientes. Sea $\boldsymbol{m}$ la captación del océano y la atmosfera es una proporción de las emisiones acumuladas que permanecen en la atmósfera después de un periodo. Sea $\boldsymbol{N}$ la población constante y las emisiones son agregados como $z_{t}=z_{t} N_{t}$ y convertido por un factor $\varnothing$ de grandezas de la emisiones en partes por millón. Con estas hipótesis el proceso de acumulación de concentración atmosférica de la polución es la siguiente:

$A_{t+1}=(1-m) A_{t}+\phi N \frac{l_{t}}{\rho}, \quad 0 \leq m \leq 1$,

debido a (49) donde $z_{t}$ ha sido sustituido por $\frac{l_{t}}{\rho}$. Se ha supuesto que $m$ es constante, pero en el último siglo el Panel Intergubernamental del cambio climático, IPCC (1994), ha calculado que la fracción en el aire de $C \mathrm{O}_{2}$ ha variádo del $15-25 \%$ en 450 PPM hasta 30-40\% en 750 PPM esto es; la captación no es independiente del nivel de emisiones, y además que existe un nivel de valor extremo en el cual deje de funcionar tal absorción, es un mecanismo conocido como umbral que se expresa así:

$$
m\left(A_{t}\right)=1-\frac{A_{t}}{\hat{A}},
$$

donde $\hat{A}$ representa el máximo de las adiciones a la concentración atmosférica para el cual ningún sumidero puede reducir la fracción de emisión, de (52) se deduce que solo es de interés estudiar el caso $A_{t}<\hat{A}$

Cuando no hay emisores debido a la actividad humana, $A_{t}=0$ el sistema natural está en equilibrio como en el nivel pre-industrial y la captación es completa, $\boldsymbol{m}=\mathbf{1}$ en este caso (51) se convierte en,

$$
A_{t+1}=\frac{A_{t}^{2}}{\hat{A}}+\emptyset N \frac{l_{t}}{\rho}, A_{t}<\hat{A}, \xi=\emptyset N .
$$


De (47) y (51) tenemos los siguientes dos modelos: (54 a ), la limpieza proposicional, o la de captura con umbral (54 b),

$$
\left\{\begin{aligned}
c_{t+1} & =f\left(l_{t}, A_{t}\right) \\
l_{t+1} & =b\left(l_{t}, c_{t}\right) \\
A_{t+1}=g\left(A_{t}\right)+\xi \frac{l_{t}}{\rho}, \quad & g_{a}=(1-m) A_{t}, \\
g_{b} & =\frac{A_{t}^{2}}{\hat{A}}
\end{aligned}\right.
$$

De (47) obtenemos el siguiente sistema (54 a) :

$$
\left\{\begin{array}{c}
c_{t+1}=l_{t}^{\frac{\beta}{\alpha}}\left(1+\frac{A_{t}}{k}\right)^{\frac{1}{\alpha}} \\
l_{t+1}=b\left(l_{t}-c_{t}\right) \\
A_{t+1}=(1-m) A_{t}+\xi \frac{l_{t}}{\rho}
\end{array}\right.
$$

cuyo origen es un punto de silla y el otro equilibrio tiene las dos primeras componentes así:

$$
\begin{array}{r}
(\bar{c}, \vec{l})=\left(\left(\frac{b-1}{b}\right)^{\frac{\beta}{\beta-\alpha}}(1+\theta(\bar{A}))^{-\frac{1}{(\beta-\alpha)}},\right. \\
\left.\left(\frac{b-1}{b}\right)^{\frac{\alpha}{\beta-\alpha}}(1+\theta(\bar{A}))^{-\frac{1}{(\beta-\alpha)}}\right)
\end{array}
$$

dejando todos los parámetros fijos excepto $\rho$, la tercera componente es $\bar{A}=\frac{\mu(\bar{A})}{2}$ con,

$$
\mu(\bar{A})=\left(\frac{\varepsilon}{\rho}\right)\left(\frac{b-1}{b}\right)(1+\theta(\bar{A}))^{-\frac{1}{\beta-\alpha}}
$$

el valor del estado de reposo de los poluentes es determinado por la intercepción de $h(\bar{A})=2 \bar{A}$ con la curva $j(\bar{A})$, el lado derecho de la ecuación (57) este estado de reposo existe porque $\boldsymbol{h}$ y $\boldsymbol{j}$ son continuas $h(0)<j(0)$ y para $A_{\text {grande }}$ se tiene

$h\left(A_{\text {grande }}\right)>j\left(A_{\text {grande }}\right)$, además este equilibrio es único porque $\boldsymbol{h}$ tiene pendiente positiva y $j(\bar{A})$ tiene pendiente negativa.

Como en (44) estudiamos la estabilidad a partir de las raíces de la ecuación característica

$P(\lambda)=\lambda^{3}+(-$ Traza $J) \lambda^{2}+a_{2} \lambda-\operatorname{Det} J=0$ esto es, $\lambda^{3}+a_{1} \lambda^{2}+a_{2} \lambda+a_{3}=0$,

donde $a_{2}=\lambda_{1} \lambda_{2}+\lambda_{1} \lambda_{3}+\lambda_{2} \lambda_{3}$

para $\lambda_{i}, i \in\{1,2,3\}$, las raíces del polinomio característico. Las bifurcaciones aparecen cuando

$P(1)=0$ para $i), P(-1)=0$ para $i$ )
$P(1)=(1-a) Q(1), Q$ polinomio cuadrático con raíces complejas y $\frac{d^{2} P}{d \lambda^{2}}(1)=0$ para iv), esta última

es una condición de transversalidad.

De lo anterior se tienen las siguientes condiciones que simultáneamente garantizan la estabilidad del equilibrio:

$$
\begin{array}{rr}
\text { (i) } & 1+a_{1}+a_{2}-a_{3}>0 \\
\text { (ii) } & 1-a_{1}+a_{2}-a_{3}>0 \\
\text { (iii) } & -a_{2}+a_{1} \cdot a_{3}-a_{3}^{2}>0 \\
\text { (iv) } & 3-a_{2}>0
\end{array}
$$

y como en (44), la condición (iii) no es verificable la cual hace que se pierda la estabilidad de $E_{1}=(0,0,0)$ en una bifurcación del tipo Neimar - Socker, cuya dinámica es localmente invariante en la variedad central, como se ilustra en la siguiente figura

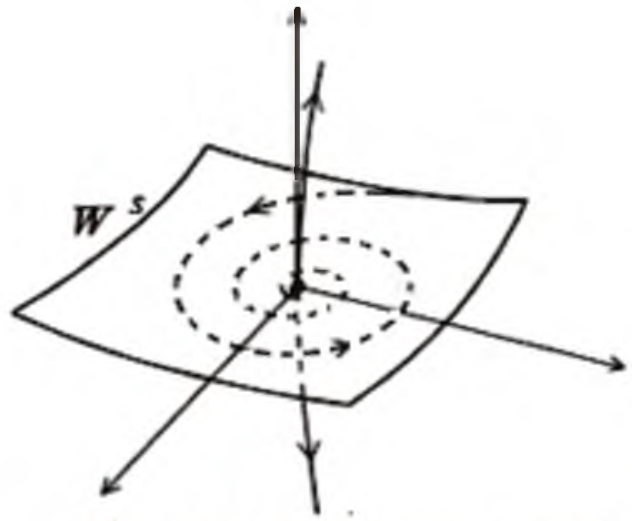

Figura 4. Bifurcación de Hopf en $\mathbb{R}^{3}$

Finalmente consideramos el sistema (54 b),

$$
\left\{\begin{array}{c}
c_{t+1}=l_{t}^{\frac{\beta}{\alpha}}\left(1+\frac{A_{t}}{k}\right)^{\frac{1}{\alpha}} \\
l_{t+1}=b\left(l_{t}-c_{t}\right) \\
A_{t+1}=\frac{A_{t}^{2}}{A}+\xi \frac{l_{t}}{\rho}
\end{array}\right.
$$

Este sistema tiene en $E_{1}=(0,0,0)$ un punto silla, además cero o dos equilibrios en el octante positivo con los siguientes dos primeras componentes,

$$
\begin{aligned}
(\bar{c}, \bar{l})= & \left(\left(\frac{b-1}{b}\right)^{\frac{\beta}{\beta-\alpha}}(1+\theta(\bar{A}))\right. \\
& \left.-\frac{1}{\beta-\alpha},\left(\frac{b-1}{b}\right)^{\frac{\alpha}{\beta-\alpha}}(1+\theta(\bar{A}))^{-\frac{1}{\beta-\alpha}}\right) .
\end{aligned}
$$


La componente $\bar{A}$ es la intercepción de las dos curvas $\boldsymbol{h}$ y $\boldsymbol{j}$ con

$$
h(\bar{A})=\hat{A} \bar{A}-\bar{A}^{2}=\frac{\hat{A} \alpha}{\beta} t_{\rho}(\bar{A})=j(\bar{A})
$$

para todos los posibles valores de $0<\rho<1$, dependiendo de $t_{\rho}$ es posible que tal intercepción no exista y para algunos valores de parámetros sea tangente.

Teniendo en cuenta las condiciones de inestabilidad (i) - (vi) para encontrar los estados de reposo no hiperbólicos tenemos que (i) siempre se cumple; cuando la intercepción entre las curvas $\boldsymbol{h}$ y $\boldsymbol{j}$ es única y no transversal, no se tiene la condición (i), es una bifurcación de tipo Fold y se puede perder la condición (iv), esto es, puede ocurrir una bifurcación de tipo Neimark-Socker.

\section{Conclusiones}

En este trabajo hemos estudiado un modelo económico que se explica mediante un sistema dinámico involucrando las variables de trabajo, capital y externalidad de la contaminación del aire causada por la actividad industrial. Hemos analizado dos modelos considerando el autocontrol de la atmosfera, proporcional o considerando la capacidad del umbral de captura; lo cual se expresa mediante un sistema de tres ecuaciones en diferencias; de ellos hemos estudiado sus equilibrios, estabilidad y bifurcaciones. En particular, se detalla las bifurcaciones de duplicación de periodos y la existencia de una órbita periódica de Hopf que aparece significativamente en estos modelos de la economía matemática y son mayor complejidad en la frontera de estas bifurcaciones, dependiendo del parámetro $\rho>0$, que es el índice de emisión de contaminante por unidad de producción. Si no traspasamos el autocontrol de la atmosfera, en la práctica significa que es posible establecer políticas para reducir el efecto invernadero.

\section{Referencias Bibliográficas}

Barrett, S. (1994). Self-enforcing international environmental agreeements. Oxford Economic Papers, 46, 878-894.

J. BENHABB AND R. H. DAY, A characterization of erratic dynamics in the overlapping generations model, J. Econ. Dynam. Control 4 , 1982.

O. J. Blanchard and S. Fischer, Lectures on Macroeconomics. Cambridge: MTT Press, 1989.

J. Crespo, T. palorcango y A. tarasyev, Dynamical systems economic Growth and the environment, springer, 2010.

D. Cao, L. Wang and Wamp; Endogenous fluctuations induced by nonlinear pollution in and OLG economy; Economic Modelling, 28 (2011) 2528 - 2531.

T. J. Conway (ed), Chapter 2. Carbon Cycle Greenhouse Gases. Climate

Manitoring and Diagnostics Laboratory, Report 27. Boulder: U.S. National Oceanic Atmospheric Adininistration, 2003.

P. A. Diamond, National debt in a neoclassical growth model, Amer. Econ. Rev. 55 (1965), 1126-1150.

Ehtamo, H., \&Hamalainen, R. P. (1993). A cooperative incentive equilibrium for a resource management problema. Journal of Economic Dynamics and Control, 17, 659-678.

R. W. Farebrother, Simplified Samuelson conditions for cubic and quartic equations. The Manchester School of Economics and Social Studies, 4:396-400, 1973.

D. GALE, Pure exchange equilibrium of dynamic economic models, J. Econ. Theory 6(1973), 12-36.

A. Medio, Chaotic Dynamics: Theory and Applications to Economics. Cambridge: Cambridge University Press, 1992.

J.M. Grandmont, on endogenous competitive busmess cycles. Econometrica, 53, 995-1045, 1985.

P.A. Samuelson, A Exact Consumption - Loan Model of interest with or withord the Social Contrivances of Money, Journal of political Economy, 66, 467-482, 1958.

P. Reichlin, Equilibrium cycles in an overlapping, Columbia University Press, N. Y, 2006.

World Economic Forum, New Growth Models, Ginebra, 2014. 